\title{
Pour une « ethnographie délinquante » : vingt ans avec les gangs au Nicaragua
}

For a "Delinquent Ethnography": Insights from Twenty Years of Gang Research in Nicaragua

\section{Dennis Rodgers}

\section{(2) OpenEdition \\ Journals}

Édition électronique

URL : https://journals.openedition.org/conflits/20235

DOI : $10.4000 /$ conflits. 20235

ISSN : $1777-5345$

Éditeur :

CECLS - Centre d'études sur les conflits - Liberté et sécurité, L'Harmattan

Édition imprimée

Date de publication : 10 octobre 2018

Pagination : $59-76$

ISBN : 978-2-343-16812-8

ISSN : 1157-996X

Référence électronique

Dennis Rodgers, «Pour une « ethnographie délinquante » : vingt ans avec les gangs au Nicaragua », Cultures \& Conflits [En ligne], 110-111 | été/automne 2018, mis en ligne le 10 octobre 2020, consulté le 08 janvier 2022. URL : http://journals.openedition.org/conflits/20235 ; DOI : https://doi.org/10.4000/ conflits.20235 


\section{Pour une « ethnographie délinquante»: vingt ans avec les gangs au Nicaragua}

\section{Dennis RODGERS}

Dennis Rodgers est professeur de recherche dans le département d'anthropologie et de sociologie de l'Institut de hautes études internationales et $d u$ développement à Genève, Suisse. Sa recherche porte sur les conflits et la violence dans les villes d'Amérique latine (Nicaragua, Argentine) et d'Asie du Sud (Inde). Il mène en particulier des recherches ethnographiques longitudinales sur la dynamique des gangs au Nicaragua depuis 1996. Il est lauréat d'une bourse ERC "Advanced Grant » pour un projet intitulé "Gangs, Gangsters, and Ganglands: Towards a Global Comparative Ethnography » (GANGS) qui développera une comparaison du phénomène des gangs au Nicaragua, en Afrique du Sud et en France (2019-2023).

T es gangs font partie d'un petit nombre de phénomènes quasi-universels, L présents au fil de l'histoire dans la plupart des sociétés à travers le monde. Tite-Live parle déjà des gangs au premier siècle avant Jésus-Christ, dans sa célèbre histoire de Rome, $A b$ Urbe Condita, et on les trouve dans des sociétés aussi diverses que les États-Unis, la France, le Kenya, le Salvador ou la Chine, parmi d'autres ${ }^{1}$. En partie à cause de cette ubiquité, il n'est pas exagéré d'affirmer que les gangs occupent une position clé dans l'imaginaire mondial de la violence. Cela fait en effet longtemps que les représentations des gangs comme une incarnation du mal, d'une sauvagerie incontrôlable, d'une barbarie brutale et insensée, perdurent dans notre conscience collective. Depuis l'étude bien-connue du journaliste Herbert Asbury, The Gangs of New York, publiée en 19272 - et transposée au grand écran en 2002 par Martin Scorsese dans un film éponyme mettant en vedette Leonardo di Caprio, Cameron Diaz et Daniel Day-Lewis - en passant par des romans tels Brighton Rock de Graham Greene (1938), La Virgen de los Sicarios de Fernando Vallejo (1994), La Mara de Rafael Ramírez Heredia (2006), ou bien Tropique de la Violence de Nathacha Appanah (2016), ainsi que des films tels que Los Olvidados

1. Hazen J. M. et D. Rodgers (eds.), Global Gangs: Street Violence across the World, Minneapolis, University of Minnesota Press, 2014.

2. Asbury H., The Gangs of New York: An Informal History of the Underworld, New York, Garden City Publishing Company, 1927. 
(1950), West Side Story (1961), The Warriors (1979), Colors (1988), Boyz $n$ the Hood (1991), Bronx-Barbés (2000), City of God (2003), Tsotsi (2005), Tribu (2007), Gangster's Paradise Jerusalema (2008), La Yuma (2009), Sin Nombre (2010), ou Black (2015), les gangs sont omniprésents autour de nous, un phénomène qui répugne et fascine en même temps.

Une raison pour cet intérêt réside dans le fait que les gangs sont des phénomènes fondamentalement révélateurs, reflétant de manière inhérente des processus sociaux essentiels tels l'exercice du pouvoir, les relations de genre, l'accumulation matérielle, la territorialité ou bien les questions identitaires, entre autres. Comme le soutient Frederic Thrasher dans son étude pionnière des gangs de Chicago dans les années 1920, ceux-ci représentent « la vie, souvent rude et farouche, mais riche en processus sociaux élémentaires importants pour tout étudiant de la société et de la nature humaine ${ }^{3} »$. Thrasher souligne ainsi que les gangs sont à la fois des phénomènes et des épiphénomènes, des institutions sociales autonomes, avec des logiques et des dynamiques internes complexes, mais aussi le reflet de structures sociales plus larges. C'est pourquoi l'étude de Thrasher n'est pas « juste » une étude des gangs de Chicago : elle ne nous offre pas seulement des informations détaillées concernant leurs caractéristiques particulières, mais dresse aussi un portrait de l'économie politique de Chicago et de la société américaine de l'époque.

Thrasher fut le fondateur d'une longue tradition de recherches ethnographiques sur les gangs qui, comme Scott Decker et David Pyrooz l'ont souligné, a produit des études parmi les plus approfondies et les plus révélatrices du phénomène à travers le monde 4 . Ceci découle en partie du fait que l'ethnographie est une approche méthodologique qui appelle au développement d'une certaine intimité avec les gangs, d'un rapprochement pour permettre au chercheur ou à la chercheuse d'interagir directement avec les gangs afin de comprendre leur logiques et dynamiques de l'intérieur. Ceci étant dit, de par sa nature particulière, l'ethnographie n'est pas une méthode nécessairement évidente à mettre en œuvre afin d'étudier les gangs. Elle soulève de nombreux dilemmes, tant pratiques qu'éthiques. Ceux-ci sont cependant souvent passés sous silence ${ }^{5}$. Cet article cherche donc à établir le début d'une conversation

3. Thrasher F., The Gang: A Study of 1313 Gangs in Chicago, Chicago, University of Chicago Press, 1927, p. 3. Texte original: «life, rough and untamed, but rich in elemental social processes significant to the student of society and human nature ».

4. Decker S. H. et D. Pyrooz, « Contemporary Gang Ethnographies », in Cullen F. T. et P. Wilcox, The Oxford Handbook of Criminological Theory, Oxford, Oxford University Press, 2011, pp. 274-293.

5. Il existe bien évidemment toute une littérature s'appliquant à développer une démarche réflexive à propos des pratiques ethnographiques dans les terrains dits «sensibles » ou « difficiles » (voir, par exemple, Lee R. M., Dangerous Fieldwork, Thousand Oaks, Sage, 1995 ; Nordstrom C. et A. C. G. M. Robben (eds.), Fieldwork under Fire: Contemporary Studies of Violence and Survival, Berkeley, University of California Press, 1995 ; Greenhouse C., E. Mertz et K. B. Warren (eds.), Ethnography in Unstable Places: Everyday Lives in Contexts of 
soutenue concernant ces questions, en me basant sur mes recherches ethnographiques sur les dynamiques des gangs au Nicaragua, entamées en 1996 et toujours en cours. À la lumière de certains faits et évènements liés à cette expérience, je plaiderai explicitement en faveur d'une " ethnographie délinquante ", déclinée de trois manières : la première centrée sur l'objet d'étude, c'est-à-dire le gang, la seconde sur le rôle particulier du chercheur ou de la chercheuse, et la troisième sur la nature même de la méthode ethnographique, surtout quand elle est déployée sur le long terme. Cette exposition mettra en exergue la réflexivité nécessaire dans l'observation des gangs, tout en soulignant la pertinence de l'ethnographie comme méthode d'enquête «transgressive », comme on le verra dans la conclusion de l'article.

\section{Gangs et ethnographie}

Qu'est-ce qui fait que l'ethnographie soit une méthode de recherche aussi féconde pour étudier les gangs ? Cela a bien évidemment à voir avec le fait que l'ethnographie implique une recherche de proximité, qui vise à étudier les individus et les communautés dans leurs espaces, dans leurs rythmes. Associée principalement à l'anthropologie et la sociologie, et particulièrement aux recherches des «écoles »-celle de Chicago dans les années 1920 et 1930 6, ou celles de Manchester dans les années 1950 et 19607 - l'ethnographie est une

Dramatic Political Change, Durham, Duke University Press, 2002 ; Bouillon F., Fresia M. et V. Tallio (dir.), Terrains Sensibles : Expériences actuelles de l'anthropologie, Paris, Centre d'études africaines, École des Hautes Études en Sciences Sociales, 2006 ; Fassin D. et A. Bensa (dir.), Les politiques de l'enquête : Épreuves ethnographiques, Paris, La Découverte, 2008). Néanmoins, très peu de celles-ci se penchent spécifiquement sur les gangs, même s'il existe une littérature extensive concernant des phénomènes connexes tels les enfants de la rue (voir, par exemple, Meunier J., Les Gamins de Bogota, Paris, Éditions Jean-Claude Lattès, 1977 ; Hecht T., After Life: An Ethnographic Novel, Durham, Duke University Press, 2006 ; Wolseth J. M., Life on the Malecón: Children and Youth on the Streets of Santo Domingo, New Brunswick, Rutgers University Press, 2014) ou le trafic de la drogue (voir, par exemple, Bourgois P., En quête de respect: Le crack à New York, Paris, Seuil, 2001 ; Contreras, R., The Stick Up Kids: Race, Drugs, Violence, and the American Dream, Berkeley, University of California Press, 2013). La plupart des études ethnographiques sur les gangs ne discutent généralement que très brièvement des problèmes pratiques et méthodologiques encourus. L'importante étude de Martin Sánchez-Jankowski, Island in the Streets: Gangs and American Urban Society (Berkeley, University of California Press, 1991) est un très bon exemple de ceci, expliquant de manière laconique et succincte que son travail de terrain n'était pas facile mais que " assez remarquablement, au cours de dix ans de recherches, je n'ai été sérieusement blessé que deux fois » (texte original : "Quite remarkably, in the more than ten years during which I conducted this research, I was only seriously injured twice »). La seule réflexion véritablement extensive concernant les dilemmes pratiques et éthiques autour de la recherche ethnographique sur les gangs est celle contenue dans la deuxième édition de l'étude classique de William Foote Whyte, Street Corner Society (Chicago, University of Chicago Press, 1955 ; la première édition est de 1943).

6. Joseph I. et Y. Grafmeyer (dir.), L'école de Chicago : Naissance de l'écologie urbaine, Paris, Les éditions du Champ Urbain, 1984 ; Chapoulie J.-M., La tradition sociologique de Chicago, 1892-1961, Paris, Seuil, 2001 ; Guth S., Chicago 1920: Aux origines de la sociologie qualitative, Paris, Téraèdre, 2004.

7. Hannerz U., Exploring the City: Inquiries Toward an Urban Anthropology, New York, Columbia University Press, 1980 ; Evens T. M. S. et D. Handelman (eds.), The Manchester School: Practice and Ethnographic Praxis in Anthropology, Oxford, Berghahn, 2006. 
méthode qui vise à s'impliquer directement dans la vie quotidienne des autres et à développer une interaction profonde et intense communément dénommée « l'observation participante ». La logique d'une telle immersion pendant une période prolongée est d'interagir de façon normative, de participer aux activités quotidiennes et d'observer les événements et les personnes dans leur contexte afin de développer une certaine intimité, un sens empathique avec l'environnement social, pour essayer d'atteindre ce que Max Weber appelait «verstehen ou la « compréhension interprétative 8 ».

Mais comment s'immerger dans l'univers des gangs ? Comme l'a observé Stephen Lyng, le caractère particulier de l'ethnographie en fait clairement une «méthode dangereuse » lorsqu'elle est mise en ouvre afin d'étudier des phénomènes violents ${ }^{9}$. Comme Antonius Robben et Carolyn Nordstrom le soulignent, « à un certain niveau, pour pouvoir discuter de la violence, les ethnologues doivent aller là où la violence a lieu, la rechercher pendant qu'elle a lieu 10 ». Ceci peut évidemment être très difficile, mais n'est clairement pas impossible, ainsi que l'attestent trois superbes études ethnographiques sur les gangs en France : Cour de Banlieue, par David Lepoutre 11, Le Capital Guerrier, par Thomas Sauvadet ${ }^{12}$, et La Formation des Bandes, par Marwan Mohammed 13. L'étude de Lepoutre explore les codes, les rites et les langages des bandes de jeunes dans la cité des Quatre-Mille à La Courneuve, celle de Sauvadet compare la concurrence et la solidarité entre jeunes dans deux cités à Paris et une à Marseille, alors que l'étude de Mohammed se focalise sur l'émergence et l'évolution des bandes dans la cité des Hautes-Noues, à Villiers-surMarne. Ces trois études nous offrent des analyses extrêmement détaillées et très fines qui vont bien au-delà des représentations médiatiques stéréotypées qui abondent à propos des gangs ou d'autres phénomènes violents ${ }^{14}$ et

8. Weber M., The Theory of Social and Economic Organization, New York, Oxford University Press, 1947.

9. Lyng S., "Dangerous methods: Risk taking and the research process ", in J. Ferrell et M. S. Hamm (eds.), Ethnography at the Edge: Crime, Deviance, and Field Research, Boston, Northeastern University Press, 1998.

10. Robben A. C. G. M. et C. Nordstrom, « The anthropology and ethnography of violence and socio-political conflict ", in C. Nordstrom et A. C. G. M. Robben (eds.), Fieldwork..., op. cit., p. 4. Texte original : " at some level, to be able to discuss violence, the ethnographer must go to where violence occurs, research it as it takes place».

11. Lepoutre D., Cour de banliene : Codes, rites et langages, Paris, Éditions Odile Jacob, 1997.

12. Sauvadet T., Le Capital guerrier: Concurrence et solidarité entre jeunes de cité, Paris, Armand Colin, 2006.

13. Mohammed M., La formation des bandes: Entre la famille, l'école et la rue, Paris, Presses Universitaires de France, 2011.

14. Comme l'a très bien indiqué Philippe Bourgois, il est essentiel que l'ethnographie de la violence ne devienne pas une "pornographie de la violence " (En quête de respect..., op. cit., p. 47). Les débats récents concernant les ouvrages de Sudhir Venkatesh (Gang Leader for a Day: A Rogue Sociologist takes to the Streets, New York, Penguin, 2008) et d'Alice Goffman (On the Run: Fugitive Life in an American City, Chicago, University of Chicago Press, 2014) démontrent néanmoins à quel point ceci est quelque chose de délicat et subjectif. C'est ce que nous soulignons avec mon collègue Gareth A. Jones dans un article récent - voir Jones G. A. et D. Rodgers, "Ethnographies and/of violence in Latin America and the Caribbean ", Ethnography, vol. 20, n 3, 2019 (à paraitre). 
incluent aussi de brèves discussions méthodologiques expliquant comment chaque chercheur a réussi à approcher les gangs étudiés.

En particulier, Lepoutre explique qu'il a enseigné pendant sept ans dans un collège de La Courneuve, où il a également vécu pendant deux ans. Cela lui a permis de tisser des liens forts avec certains individus et familles qui lui ont servi d'intermédiaires afin de s'approcher de membres de gangs. Pour sa part, Sauvadet raconte qu'il a passé une partie de sa jeunesse dans une des deux cités parisiennes étudiées. Il y était donc connu et avait un réseau préexistant. Dans l'autre cité parisienne, il emménagea "dans un petit pavillon ouvrier situé juste en face ", où l'un de ses voisins le mit en contact avec ce qu'il appelle des « informateurs-médiateurs » qui lui permirent de s'intégrer socialement. Par contre, "le terrain marseillais s'est ensuite imposé en raison des liens et de l'expérience qui m'y rattachent : j'y ai des amis à proximité et j'y passe mes vacances depuis plus d'une quinzaine d'années ». L'un de ses amis d'adolescence animait une association de boxe dans la cité et était donc crédité « d'un certain respect 15 ». Mohammed, pour sa part, a lui aussi passé sa jeunesse dans la cité des Hautes-Noues où il a effectué sa recherche - "période durant laquelle j'ai été confronté (parfois impliqué) avec ce que j'ai appelé les “embrouilles de cité" ${ }^{16} \ldots$ » - et il y est retourné plus tard afin d'y fonder une association locale avant de devenir vacataire dans la maison de quartier.

Ces trois exemples soulignent l'importance de la proximité et la nécessité d'intermédiaires afin d'approcher le gang pour l'étudier. Ceci étant dit, aucun de ces trois ethnologues ne nous offre beaucoup de détails concernant les activités d'observation participative qui leur ont permis de comprendre les gangs qu'ils ont étudiés, mis à part expliquer qu'ils ont tous noué des relations privilégiées avec certains individus, dont des membres de gangs (Lepoutre note aussi qu'il pratique un niveau élevé de boxe thaillandaise, ce qui suscite peutêtre plus de questions que de réponses...). Comme l'a souligné Jeffrey Sluka, il existe clairement une certaine réticence universelle parmi les ethnologues de la violence à parler ouvertement des détails pratiques de leurs recherches car celles-ci impliquent presque inévitablement de grands dilemmes moraux et éthiques ${ }^{17}$. Dans le cas des études sur les gangs, ceux-ci découlent principalement du fait que l'ethnographie exige une interaction rapprochée avec des gangs et des membres de gangs impliqués dans des activités illégales. Au-delà, l'extension logique de la dynamique générale de l'ethnographie en tant que méthodologie tendrait même pratiquement à pousser l'ethnologue des gangs à la délinquance, vu que l'approche demande une immersion et une participation dans les activités du phénomène étudié. Ceci est une transgression éthicomorale qui peut bien évidemment poser problème.

15. Sauvadet T., Le Capital guerrier..., op. cit., pp. 30-32.

16. Mohammed M., La formation des bandes..., op. cit., p. 423.

17. Sluka J. A., « Managing Danger in Fieldwork with Perpetrators of Political Violence and State Terror », Conflict and Society, vol.1, n 1, 2015, pp. 109-124. 
C'est d'ailleurs peut-être pour cela que l'une des meilleures descriptions des dynamiques des gangs en France publiée ces vingt dernières années est le livre de Lamence Madzou, J'étais un chef de gang ${ }^{18}$. Cet ouvrage est le récit autobiographique - édité et commenté par la sociologue Marie-Hélène Bacqué - de Madzou, qui fut chef des «Fight Boys », une bande de jeunes dans la cité Montconseil à Corbeil-Essonnes. Le fait que Madzou était membre de gang lui donne bien évidemment un regard privilégié sur le phénomène et lui permet d'expliquer la logique et les dynamiques de «son » gang, car il a été centralement impliqué dans les activités délinquantes de celui-ci. Ceci étant dit, peu d'anciens membres de gangs écrivent à propos de leurs expériences, et encore moins de manière académique, même s'il faut bien noter que certains deviennent universitaires, comme par exemple Robert Durán, professeur à l'université du Texas A\&M aux États-Unis, et auteur de Gang Life in Two Cities ${ }^{19}$, une étude comparative des gangs à Denver dans le Colorado et à Ogden en Utah, où il fut membre d'un gang dans sa jeunesse, ou bien Randol Contreras, professeur à l'université et auteur de l'excellente étude The Stick-Up Kids ${ }^{20}$, à propos d'un gang de braqueurs de trafiquants de drogue dans le Bronx à New York, avec lequel il a eu des liens quand il était jeune. Il y a aussi des anthropologues qui deviennent membre d'un gang - c'est ce qui m'est arrivé dans le cadre des recherches que j'ai effectuées au Nicaragua...

\section{Ethnologue-gangster au Nicaragua}

Le Nicaragua est un pays d'Amérique centrale, qui compte six millions d'habitants - plus une diaspora d'un million hors du pays - dont presque un tiers est concentré à Managua, la capitale. Deuxième pays le plus pauvre de l'hémisphère ouest après Haïti, le Nicaragua est surtout connu pour la révolution Sandiniste qui, en 1979, renversa la plus longue dictature de toute l'histoire latino-américaine, celle de la famille Somoza qui cumula presque cinq décennies au pouvoir ${ }^{21}$. Cette révolution populaire promulgua toute une série de programmes sociaux pendant les années 1980 et fit rêver beaucoup de monde, tant au Nicaragua qu'à l'étranger. L'accession au pouvoir des Sandinistes engendra cependant une guerre civile brutale entre le nouveau régime révolutionnaire et les forces de la Contra soutenues par les ÉtatsUnis ${ }^{22}$, qui ne prit fin qu'en 1990, suite à la défaite électorale des Sandinistes (qui reste le seul parti révolutionnaire en Amérique latine à avoir rendu le pouvoir pacifiquement suite à une défaite électorale). Le Nicaragua est depuis lors rentré dans le rang, appliquant avec diligence les politiques néolibérales

18. Madzou L. et M.-H. Bacqué, J'étais un chef de gang, Paris, La Découverte/Poche, 2008.

19. Durán R. J., Gang Life in Trwo Cities: An Insider's Journey, New York, Columbia University Press, 2013.

20. Contreras R., The Stickup Kids: Race, drugs, violence and the American Dream, Berkeley, University of California Press, 2013.

21. Rosset P. et J. Vandermeer (eds.), The Nicaragua Reader: Documents of a Revolution under Fire, New York, Grove Press, 1983.

22. Harrison P., États-Unis contra Nicaragua, Genève, Centre Europe-Tiers Monde, 1988. 
du Consensus de Washington, s'appauvrissant toujours plus d'année en année, à tel point qu'il fut l'un des trois bénéficiaires latino-américains du programme des Pays Pauvres Très Endettés ${ }^{23}$. Le pays est aujourd'hui contrôlé par une élite oligarchique - dont font maintenant partie beaucoup de dirigeants historiques du sandinisme - qui semble n'avoir d'autre objectif que d'assurer son bien-être et le maintien de son train de vie luxueux au détriment du développement économique et social du reste de la population. Ainsi que l'ont fait remarquer de nombreux spécialistes de la région, le seul point positif de la trajectoire postrévolutionnaire du pays est de ne pas avoir sombré dans une nouvelle guerre civile depuis 1990, point de vue optimiste si l'on considère les troubles secouant le pays depuis avril $2018{ }^{24}$.

La révolution sandiniste fut un des points de repère politique les plus importants de mon adolescence. C'est pour cela que je suis allé au Nicaragua pour la première fois en juillet 1996. Bien qu'ayant six ans de retard, j'y cherchais les traces de la révolution qui m’avait tant inspiré... Je ne suis donc pas allé au Nicaragua afin d'y étudier les gangs, dont je ne suspectais alors pas l'existence. La violence était un thème qui ne m'intéressait pas. Mon projet de thèse de doctorat se focalisait sur la relation potentielle entre les idéaux révolutionnaires et les stratégies de survie des pauvres, et j'espérais essentiellement découvrir une corrélation entre les convictions politiques de gauche et le recours à des stratégies basées sur la solidarité et la coopération d'une part, et les convictions politiques de droite avec l'entreprise individuelle, d'autre part. Ce fut donc un énorme choc de réaliser, rapidement après mon arrivée au Nicaragua, qu'il restait très peu des idéaux de la révolution sandiniste, tout comme de constater que la violence constituait une caractéristique importante de la réalité quotidienne du Nicaragua, plus particulièrement sous la forme des gangs, dénommés localement «pandillas».

Trois jours après mon arrivée au Nicaragua, des membres d'un gang m’ont attaqué et tabassé. Puis je subis assez régulièrement d'autres épisodes de ce genre au cours des deux premiers mois de mon séjour, notamment parce que j'allais régulièrement visiter des quartiers pauvres seul, afin d'essayer d'en trouver un où m'établir pour y développer mes recherches ethnographiques. Cette violence inattendue a été très traumatisante - je n'en avais jamais fait l'expérience auparavant - et $j$ 'ai failli quitter le Nicaragua plusieurs fois lors de ces deux premiers mois. En même temps, cette violence a aussi profondément affecté ma recherche, dans la mesure où elle me força à interagir avec - et à prêter attention à - un phénomène dont je ne me souciais absolument pas mais qui était clairement un élément très important de la réalité sociale nicara-

23. Walker T. W. (ed.), Nicaragua without Illusions: Regime Transition and Structural Adjustment in the 1990s, Wilmington, Scholarly Resources Inc, 1997.

24. Collombon M. et D. Rodgers, «Sandinismo 2.0 : Reconfigurations autoritaires du politique, nouvel ordre économique, et conflit social ", Cahiers des Amériques, vol. 87, n 1, 2018, pp. 13-36. 
guayenne. De ce point de vue, cette expérience exemplifie la nature dialectique de l'ethnographie, et le fait qu'il est impossible de mettre en œuvre une telle approche méthodologique sans s'y soumettre, dans la mesure où l'immersion radicale dans un contexte particulier, qui est à la base de l'ethnographie, ne permet pas de se voiler la face et d'ignorer ce qui se passe autour de nous. Aussi, lorsque je pus enfin emménager dans un quartier pauvre, deux mois après mon arrivée au Nicaragua - j'avais séjourné jusque-là chez un professeur d'une université locale - les gangs et leur violence étaient devenus le sujet principal de ma recherche.

Le quartier dans lequel j'ai emménagé - et auquel je retourne régulièrement depuis maintenant plus de vingt ans - est le barrio Luis Fanor Hernández (ce nom est bien évidemment fictif, afin de protéger l’identité du quartier et la vie privée ainsi que la sécurité de ses habitants). Celui-ci jouissait alors d'une réputation particulièrement sulfureuse à cause de son gang, à l'époque l'un des plus notoires du district. Quelques semaines seulement après m’être établi dans le barrio Luis Fanor Hernández, je fus initié au sein du gang du quartier. Cette initiation était dans une certaine mesure presque naturelle : j'étais jeune - 23 ans à l'époque - homme, et du fait de la mise en œuvre d'une enquête ethnographique, je traînais dans les rues du quartier, donc dans un espace privilégié du gang... Il est clair que le débarquement au cour du gang d'un étranger, chercheur de surcroît, peut paraître rocambolesque et invraisemblable. Cette expérience a été présentée dans le détail ailleurs ${ }^{25}$, néanmoins on peut dire qu'un facteur important, compte tenu des dynamiques en cours, a été la question de la réputation du gang, elle-même découlant en grande partie des caractéristiques de ses membres individuels. Le gang décida de m’intégrer car cela lui permettait de devenir le seul gang de Managua à avoir un «chele pandillero », ou membre étranger, et vit sa notoriété s'accroître de manière significative. Pour ma part, j'acceptais de devenir membre du gang pour une raison très simple, l'idée selon laquelle « si vous ne pouvez pas les vaincre, joignez-vous à eux ». En d'autres termes, j'ai rejoint le gang comme stratégie de survie plutôt que comme stratégie de recherche.

Devenir membre du gang m'a bien évidement fourni une opportunité de recherche ethnographique incroyable ${ }^{26}$. J'ai pu effectuer de l'observation participante au sein du groupe, passant énormément de temps à traîner dans les rues du quartier, fumant, buvant et vannant avec les autres membres du gang. J'ai participé à nombre de leurs activités, tant violentes que non-violentes, et j'ai pu me familiariser ainsi avec les codes, rites et pratiques du gang. J'ai passé beaucoup de temps individuellement avec des membres de gangs, tant dans la

25. Rodgers D., "Joining the gang and becoming a broder: The violence of ethnography in contemporary Nicaragua ", Bulletin of Latin American Research, vol. 27, n 4, 2007, pp. 444461.

26. Il est important de préciser que je n'ai jamais caché mon rôle de chercheur aux membres du gang, qui semblent l'avoir accepté comme condition de mon intégration. 
rue que chez eux, à leur demander ce qui les avait poussés à se joindre au gang, savoir comment ils se voyaient, ce qu'ils pensaient des autres... J'ai pu comparer leurs discours avec leurs pratiques et les observer agir et interagir dans diverses circonstances, dont beaucoup qui auraient été impossibles pour quelqu'un qui n'était pas membre du gang. D’une manière plus générale, mon immersion dans le gang m'a permis de développer ce que Loïc Wacquant a dénommé dans la préface de l'édition américaine de sa célèbre étude sur la boxe, Corps et âme, une " sociologie charnelle ", c'est-à-dire impliquant une « conversion morale et sensuelle au contexte de recherche 27 ».

J'ai bien évidemment dû faire face à différents dilemmes moraux et à des situations compliquées à gérer, que je décris en détail dans des écrits précédents ${ }^{28}$, soulignant en particulier que les décisions morales se prennent souvent de manière très situationnelle et qu'à certains niveaux l'ethnologue ne peut qu'inévitablement devenir complice. Que faire lorsque l'on découvre que des individus faisant parti de notre champ de recherche ont commis des crimes, ce qui est presque inévitable lorsque l'on étudie les gangs ? Faut-il les dénoncer, les confronter ou bien les ignorer ? Les trois options impliquent différentes complicités - avec les autorités, avec le gang, avec l'individu. Elles doivent clairement être considérées par rapport à un cadre éthique plus large, et par rapport à la nature transgressive inhérente à l'ethnographie, mais en fin de compte elles engagent toujours des choix avec lesquels beaucoup ne seront pas d'accord. Au final, comme l'a très bien dit William Foote Whyte, le critère déterminant est que «l'ethnographe [...] doit continuer à vivre avec lui-même. S’il se trouve en train de participer à des activités qu'il [...] perçoit [comme] immorales, il est probable qu'il commencera à se demander quel genre de personne il est 29 ».

Ces expériences transgressives ont aussi été au cœur de ma thèse de doctorat ${ }^{30}$, dont le titre, "Vivre à l'ombre de la mort », reprenait une expression que les membres du gang utilisaient fréquemment pour décrire de manière très viscérale leur condition sociale - «somos muerte arriba » - mais jouait dessus afin de caractériser non seulement l'univers des gangs mais aussi la condition contemporaine du Nicaragua, argumentant que les gangs constituaient une stratégie de « survie sociale » face à des conditions structurelles de pauvreté,

27. Wacquant L., Body and Soul: Notebooks of an Apprentice Boxer, Oxford, Oxford University Press, 2004, pp. viii \& vii. En français, voir Corps et âme : Carnets ethnographiques d'un apprenti boxeur, Paris, Agone, 2001.

28. Rodgers D., "Joining the gang...», op. cit.

29. Whyte W. F., Street Corner Society, Chicago, University of Chicago Press, 1955, p. 327. Texte original : "the field worker [...] has to continue living with himself. If the participant observer finds himself engaging in behavior that be [...] think $[\mathrm{s}]$ of as immoral, then be is likely to begin to wonder what sort of a person be is ".

30. Rodgers D., Living in the Shadow of Death: Violence, Pandillas and Social Disintegration in Contemporary Urban Nicaragua, thèse de doctorat, département d'anthropologie sociale, Université de Cambridge, 2000. 
d'exclusion, et d'inégalités correspondant à une "mort sociale 31 ». Le fait d'être devenu membre du gang du barrio Luis Fanor Hernández m'a aussi permis d'engager les recherches ethnographiques longitudinales que je poursuis depuis maintenant plus de vingt ans. Bien qu'ayant pris ma « retraite » du gang à mon départ du Nicaragua, en juillet 1997, suite à mon premier séjour, les relations que j'y ai nouées ont perduré et je reste un «vieux de la vieille » respecté par les anciens et nouveaux membres du gang. Ces derniers ont donc toujours été prêts à me parler, à répondre à mes questions, à partager des informations concernant leurs activités, tant légales qu'illégales, lors de mes retours au barrio Luis Fanor Hernández en 2002, 2003, 2007, 2009, 2012, 2014 et 2016 .

\section{La recherche ethnographique longitudinale}

La nature longitudinale de mes recherches a été très importante pour ma compréhension des gangs nicaraguayens. Trop de recherches sur les gangs même ethnographiques - se résument à ce que nous pourrions appeler un portrait «Polaroid », c'est-à-dire saisissant un moment figé dans le temps. Ainsi que l'a bien noté John Hagedorn, les gangs sont des institutions souvent éphémères, elles existent en mouvement, à tel point que « le gang d'aujourd'hui peut devenir un cartel de drogue demain, puis se transformer en milice ethnique le jour suivant 32 ». Si nous ne saisissons pas ce mouvement, notre compréhension des gangs sera inévitablement limitée, comme le démontre l'évolution du gang dans le barrio Luis Fanor Hernández au cours des vingt dernières années. Comme je l'ai décrit en détail ailleurs ${ }^{33}$, l'évolution institutionnelle du gang du barrio Luis Fanor Hernández a suivi une trajectoire qui l'a vu se transformer d'une bande d'anciens conscrits organisée autour de la défense de leurs familles et amis au début des années 1990, à l'institutionnalisation de cette éthique particulière autour d'une identité territoriale de quartier au milieu des années 1990, à un gang de trafiquants de drogue au début des années 2000, à l'élimination du gang suite à la professionnalisation du trafic de drogues et l'émergence d'un groupe criminel organisé connu sous le nom de

31. Rodgers D., "Living in the shadow of death: Gangs, violence, and social order in urban Nicaragua, 1996-2002 ", Journal of Latin American Studies, vol. 38, n² 2, 2006, pp. 267-292 ; Rodgers D., "Les bandes comme stratégie de "survie sociale" au Nicaragua », in Bataillon G. et D. Merklen (dir.), L'Expérience des Situations-Limites, Paris, Karthala, 2009.

32. Hagedorn J. M., A World of Gangs: Armed Young Men and Gangsta Culture, Minneapolis, University of Minnesota Press, 2008, p. xxv. Texte original : «today's youth gang might become a drug posse tomorrow, even transform into an ethnic militia or a vigilante group the next day".

33. Rodgers D., "Living in the shadow...», op. cit. ; Rodgers D., "When vigilantes turn bad: Gangs, violence, and social change in urban Nicaragua », in Pratten D. et A. Sen (eds.), Global Vigilantes, London, Hurst, 2007 ; Rodgers D., " Nicaragua's gangs: Historical legacy or contemporary symptom? ", NACLA Report on the Americas, vol. 45, $\mathrm{n}^{\circ}$ 1, 2012, pp. 66-69; Rodgers D., "The Moral Economy of Murder: Violence, Death, and Social Order in Gangland Nicaragua », in Auyero J., Bourgois P. et N. Scheper-Hughes (eds.), Violence at the Urban Margins, Oxford, Oxford University Press, 2015, pp. 21-40. 
《 cartelito » à la fin des années 2000 , à la réémergence organique de gangs d'adolescents au début des années 2010 suite à la chute du cartelito, à la répression de ces gangs de rue à travers de nouvelles formes de police au milieu de l'année 2010 et leur remplacement par des gangs déterritorialisés de femmes.

Cette évolution particulière du gang du barrio Luis Fanor Hernández est liée tant à des facteurs endogènes qu'exogènes, et implique des changements aux dynamiques internes du groupe (transformation de la logique de la bande du social à l'économique, déterritorialisation de la bande ${ }^{34}$ ), la mondialisation (et le passage à grande échelle de la drogue à travers le Nicaragua à partir de 1999 35), l'économie politique de l'espace «sociologique » du quartier (son occupation par le cartelito et sa recherche d'un monopole sur la violence ${ }^{36}$ ), ou bien encore la répression étatique (en particulier par un État cherchant à contrôler tant les populations de quartiers pauvres que le discours concernant la sécurité au Nicaragua ${ }^{37}$ ). Elle est clairement complexe et souvent difficile à décrire de manière cohérente, mais surtout, elle est impossible à comprendre sans une perspective longitudinale, c'est-à-dire sans une perspective de longue durée. Ceci est vrai tant au niveau de mes recherches que de l'expérience vécue des membres du gang du quartier. Je suis même devenu une sorte de « mémoire vivante » du gang du barrio Luis Fanor Hernández, à tel point que lors de mes deux dernières visites en 2014 et 2016, j'ai donné des petites conférences impromptues à propos de l'histoire du gang dans le quartier et de son évolution jusqu'aux membres actuels, car la transmission des connaissances fut interrompue par la domination du cartelito pendant la deuxième moitié des années 2000.

C'est notamment pour éviter ce genre de «trous de mémoire » que je retourne régulièrement au barrio Luis Fanor Hernández, afin d'y continuer mes recherches ethnographiques, même s'il faut admettre que la nature de celles-ci a changé au cours des vingt dernières années. J'effectue moins d'observation participante au sein du gang et je participe moins à ses activités par rapport à mes deux premières visites en 1996-1997 et 2002. Cette évolution dans mes pratiques de recherche est liée à plusieurs facteurs. Premièrement, les durées de mes voyages au Nicaragua sont généralement plus courtes depuis mon séjour initial d'un an en 1996-97. La plus longue depuis - en 2002 - a duré trois mois, la plus courte - en 2016 - deux semaines. La durée moyenne de mes visites depuis 1996-97 est d'un mois, et ceci rend l'observation participante de certains types d'événements et de comportements - ceux qui reposent sur un engagement répété à long terme - difficile ${ }^{38}$. Deuxièmement, je suis également devenu de plus en plus réticent à adopter les comportements à

34. Rodgers D., "Living in the shadow...", op. cit.

35. Rodgers D., « Drug booms and busts: Poverty and Prosperity in a Nicaraguan Narco-barrio », Third World Quarterly, vol. 39, n² 2, 2018, pp. 261-276.

36. Rodgers D., «The moral economy of murder...», op. cit., pp. 21-40.

37. Collombon M et D. Rodgers, «Sandinismo 2.0... », op. cit. 
risque associés à l'observation participante au sein d'un gang ${ }^{39}$. D'une part, cela est dû au fait que, en vieillissant, je suis devenu (un peu) plus sage. D'un autre côté, ceci peut aussi être lié à l'évolution des activités de base des membres du gang, dont par exemple leur consommation de drogues. Quand je suis arrivé au barrio Luis Fanor Hernández pour la première fois, la drogue la plus répandue était la marihuana, mais celle-ci fut remplacée à partir du début des années 2000 par la consommation de crack. Ceci rendit les membres du gang non seulement plus imprévisibles, compliquant parfois mon interaction avec eux, mais le fait que - contrairement à la marihuana pendant les années 1990 je n'ai pas participé à la consommation de crack, me distancia socialement de certains membres du gang ${ }^{40}$.

Ceci n’a pas nécessairement été un désavantage, car cela me permit de développer des nouveaux angles et perspectives de recherche. En particulier, au fil des années, je me suis de plus en plus concentré sur la réalisation d'entretiens individuels ciblés avec des anciens membres du gang. J'ai notamment commencé à répéter les entretiens avec certains individus, afin de retracer leurs parcours de vie. Outre des entrevues formelles avec cinquante-huit membres de gangs du barrio Luis Fanor Hernández entre 1996 et 2016, j’ai aussi interviewé à de multiples reprises un groupe de dix-huit membres (dont sept chaque fois depuis ma première visite en 1996-97 ; deux chaque fois depuis 2002, deux depuis 2003, deux depuis 2007, trois depuis 2009, une depuis 2012, puis une autre personne depuis $2016^{41}$ ). Ce groupe de personnes a été sélectionné à travers une combinaison de hasard, de chance et d'échantillonnage ciblé, et offre une série de trajectoires de vies « archétypales » des membres du gang du barrio Luis Fanor Hernández ${ }^{42}$. Ces dix-huit histoires de vie sont fondamentalement longitudinales, et m'ont été particulièrement utiles afin de développer une analyse plus dynamique et nuancée des pratiques sociales dans

38. Le boom des moyens de communication des vingt dernières années, dont en particulier ceux liés à l'Internet tels le courriel et les réseaux sociaux, a aussi introduit un autre élément d'observation participante à ma recherche et aussi changé la manière dont ma recherche s'effectue. Cf. Rodgers D., «From "broder” to “don”: Methodological Reflections on Longitudinal Gang Research in Nicaragua », in Koonings K., Kruijt D. et D. Rodgers (eds.), Ethnography as Risky Business: Field Research in Violent and Sensitive Contexts, Lanham, Lexington Books, 2019, (à paraître). Pour une discussion plus large de la manière dont les moyens de communication liés à l'Internet peuvent affecter le travail ethnographique dans un contexte dangereux, voir Käihkö I., "Conflict Chatnography: Instant Messaging Apps, Social Media and Conflict Ethnography in Ukraine », Ethnography, 2018, https://doi.org/10.1177/ 1466138118781640.

39. Rodgers D., "Joining the gang...», op. cit.

40. La consommation du crack était une activité individuelle, alors que la consommation de marihuana pendant les années 1990 était une activité collective pour le gang, ce qui joua un rôle important dans l'établissement de relations de confiance avec les membres du gang lors de mon premier séjour dans le barrio Luis Fanor Hernández.

41. J'ai également interrogé huit autres membres de gangs à plusieurs occasions, quoique plus irrégulièrement. À ces entrevues formelles s'ajoutent onze entrevues de groupe, ainsi que des centaines d'heures de conversation et d'interaction informelles avec des membres du gang passé et présent, ainsi qu'une centaine d'entrevues à propos du gang avec des habitants du barrio Luis Fanor Hernández. 
Elles m’ont également permis de constater ce qui arrive aux membres du gang une fois qu'ils le quittent. Ceci est particulièrement intéressant car on observe une tendance assez nette qui est que le fait d'avoir été membre du gang semble améliorer les chances d'un jeune de s'en sortir économiquement dans le quartier, en tout cas comparé à ceux qui n’ont pas été membres du gang. Prenez par exemple Bismarck, qui fut membre du gang dans les années 1990, puis se convertissait en trafiquant de drogue pendant 6 ans au début des années 2000, puis est sorti du trafic de drogue, investissant ses bénéfices et devenant le plus grand propriétaire foncier du quartier dans les années 2010, s'enrichissant au point de pouvoir se payer un pontage gastrique dans l'hôpital le plus exclusif de Managua en 2013 43. Ou bien Milton, qui fut aussi membre du gang dans les années 1990, puis trafiquant de drogue pendant un an au milieu des années 2000, et qui a utilisé certaines compétences apprises lors de cette dernière expérience afin de développer une fabrique de vente de tortilla basée sur un modèle de production et de livraison « juste à temps » qui lui ont permis de devenir le plus grand producteur de tortilla du district et de bien gagner sa vie au point de pouvoir ajouter un deuxième étage à sa maison, chose rare dans les quartiers pauvres de Managua soumis aux risques de secousses sismiques ${ }^{44}$.

\section{L'histoire de Pablo Escobar dans le barrio}

Ceci étant dit, ainsi que l'a noté Christian Lund, «il est plus facile d'établir la cohérence de son approche méthodologique après coup, car les détours inutiles peuvent alors être ignorés ou même effacés afin de la rendre plus propre qu'elle ne l'était en cours de recherche $45 »$. Ceci est particulièrement vrai

42. Ma notion de trajectoire « archétypale " s'inspire (plus ou moins) de l'étude classique de Thomas Belmonte (The Broken Fountain, New York, Columbia University Press, 1979) sur le monde criminel napolitain à la fin des années 1960 et au début des années 1970, dans laquelle il s’inspire de la notion jungienne de « l'archétype »-l'idée qu'il existe des modèles cognitifs fondamentaux à travers lesquels « l'inconscient collectif » comprend le monde - afin de conceptualiser la façon dont la vie sociale dans les bas-fonds napolitains était caractérisée par un nombre limité de rôles "protéiformes », telles le "Fripon » ou le "Bouc émissaire ", par exemple. L'étude de Belmonte propose une série de portraits détaillés d'individus correspondant à différents archétypes, afin d'appréhender la dynamique sous-jacente plus large du monde dans lequel ces individus vivent. Â ce titre, la notion d'archétype est illustrative plutôt que représentative, mais elle constitue un outil heuristique particulièrement utile afin de penser les situations où les trajectoires de vie des membres d'un groupe tendent à se conformer à un nombre limité d'options, comme c'est le cas d'un gang. Sur cette question, voir aussi Brenneman R., Homies and Hermanos: God and Gangs in Central America, Oxford, Oxford University Press, 2011.

43. Rodgers D., "Critique of Urban Violence: Bismarckian Transformations in Contemporary Nicaragua ", Theory, Culture, and Society, vol. 33, n 7-8, 2016, pp. 85-109.

44. Rodgers D., "Of Drugs, Tortillas, and Real Estate: On the Tangible and Intangible Benefits of Drug Dealing in Nicaragua ", in Arias E. D. et T. Grisaffi (eds.), Cocaine's Products: The Governance of the Global Narcotics Trade, Durham, Duke University Press, 2019 (à paraître). 
de la recherche longitudinale qui peut aussi donner lieu à certains problèmes. En particulier, une approche longitudinale peut prodiguer à l'ethnologue une confiance excessive, un sens d'omniscience. J'y ai été confronté lors de ma visite du barrio Luis Fanor Hernández en 2014, à l'occasion d'un entretien avec un membre du gang appelé Bayardo. Nous étions en train de parler de l'histoire du trafic de drogue dans le barrio Luis Fanor Hernández et j'essayais de lui démontrer - pour des raisons que je dois admettre plutôt vaniteuses oh combien je la connaissais bien, lui expliquant comment ce trafic avait ses origines dans le trafic de marihuana d'un individu appelé el Indio Viejo pendant les années 1990 avant que celui-ci ne passe au trafic de cocaïne au début des années 2000, après quoi el Indio Viejo constitua le cartelito et s'impliqua dans le trafic international vers la fin des années 2000 46. Bayardo écouta mes vantardises pendant un bout de temps puis m'interrompit de manière un peu exaspérée :

«-Pues, Dennis, tu sais que la cocaïne n'est rien de nouveau ici dans le barrio ? El Indio Viejo n'était pas le premier à en amener ici.

-Ouais, d'accord, t'as raison, c'est vrai, j'ai oublié la fois ou nous avions braqué ce diplomate en 1997 et qu'il avait plein de cocaïne avec lui...

-Non, non, Dennis, c'était il y a bien plus longtemps que ça, il y avait de la cocaïne ici dans le barrio dans les années 1980, et c'est Pablo Escobar, tu sais, le poderoso colombien, qui l'a amenée.

-Quoi ? No jodas, maje, je sais qui est Pablo Escobar, mais il n'est jamais venu ici au barrio, fais pas chier, mec, solo mate sós !

-Non, non, Dennis, c'est vrai, je te dis, je l'ai vu de mes propres yeux ! Mais j'étais un gamin à l'époque, donc je ne me souviens pas très bien, tu devrais plutôt aller parler au viejo René Vargas, lui il pourra tout te raconter, parce que le mec est resté chez lui...»

Je partis bien sûr immédiatement à la recherche de Don René, qui me raconta une histoire absolument ahurissante :

"Oui, c'est vrai, Pablo Escobar a séjourné ici au barrio dans les années 1980. Je pense que c'était en 1984-85, quelque chose comme ça. Il est resté chez ma mère, elle louait des chambres, et un jour des gens du gouvernement sont venus et lui ont demandé si elle pouvait héberger quatre personnes. Elle a dit oui, pas de problème, et donc Pablo Escobar est arrivé, avec un autre colombien qui s'appelait Gustavo Gaviria, ainsi qu'un Salvadoreño, Raul Mata, et un mexicain, cómo se llamaba, algo Gacha (comment est-ce qu'il s'appelait,

45. Lund C., "Of What is This a Case? Analytical Movements in Qualitative Social Science Research ", Human Organization, vol. 73, n 3, 2014, p. 231.

46. Rodgers D., « Drug booms and busts... », op. cit. 
quelque chose Gacha)... Ils sont restés plusieurs semaines, et payaient super bien, et sont devenus super amis avec les gens du barrio, payant des tournées et tout le reste. Je buvais avec eux presque tous les jours, mais la personne avec qui tu devrais vraiment parler c'est Lucia, tu sais, la suegra (la belle-mère) de ton pote Julio avec qui je te vois tout le temps - ella bailó por el narco (elle a dansé pour le trafiquant - dans ce contexte, un euphémisme pour dire qu'elle était prostituée). »

Je décidai qu'il était peut-être mieux de ne pas essayer de parler directement avec Lucia, mais je demandai à sa fille, Marlene, l'épouse de mon ami Julio, si c'était vrai que sa mère avait connu Pablo Escobar. Celle-ci me répondit sans hésiter que oui, que c'était absolument vrai, et qu'en plus elle avait couché avec lui quand elle était prostituée et qu'elle s'en vantait toujours après avoir bu quelques verres de trop. Cette confirmation apparente nonobstant, je dois dire que je n'étais pas du tout convaincu que Pablo Escobar était vraiment passé par le barrio Luis Fanor Hernández; il me semblait bien plus probable que cette personne était en fait un colombien quelconque, qui du fait du peu de visites d'étrangers au quartier, ainsi que de la notoriété de Pablo Escobar à l'époque, avait été associé à ce dernier dans la mémoire des gens. J'ai donc demandé à Marlene si elle serait prête à montrer une photo de Pablo Escobar à sa mère, sans lui dire qui c'était, et en lui demandant si elle le reconnaissait. Elle acquiesça et je suis donc immédiatement allé à un cyber-café trouver une photo de Pablo Escobar sur l'internet que j'imprimais et lui donnais le soir même.

Le lendemain Marlene est venu me voir et me raconta que sa mère s'était écriée : «Ay, mi Pablito lindo » en voyant la photo, le couvrant de baisers, et confirmant que c'était bien lui la personne qu'elle avait connu dans le barrio dans les années 1980. Maintenant complètement abasourdi, je me mis à effectuer des recherches un peu plus approfondies à propos de Pablo Escobar. Celles-ci révélèrent en premier lieu que tous les noms mentionnés par Don René - Gaviria, Mata, Gacha - étaient effectivement des noms d'associés connus d'Escobar. Mais encore plus surprenant, je découvris que Pablo Escobar était bel et bien venu au Nicaragua en 1984 - en tout cas d'après Barry Seal, l'agent de l'Administration de la lutte antidrogue américaine (ou DEA, Drug Enforcement Administration) chargé de le traquer, et qui prit une photo célèbre de lui - accompagné du Mexicain Rodriguez Gacha - à l'aéroport de Managua le 25 juin de cette année-là 47. D'après l'autobiographie du fils d'Escobar ${ }^{48}$, celui-ci est en fait allé plusieurs fois au Nicaragua pendant les années 1980, cherchant à négocier un accord avec le gouvernement sandiniste

47. Voir : http://www.proyectopabloescobar.com/2011/05/pablo-escobar-en-nicaragua.html (consulté le 19 novembre 2018).

48. Escobar J. P., Pablo Escobar: Mi Padre, Bogotá, Planeta Colombia, 2014. 
de l'époque afin de lui permettre de passer ses cargaisons de drogue à travers le pays (ce que les Sandinistes refusèrent, mais en même temps, ils ne firent rien pour arrêter Escobar, ne voulant pas le fâcher vu la nature violente de ses relations avec l'État colombien...). Ce serait assez logique qu'Escobar n'ait pas voulu rester dans un hôtel cinq étoiles lors de ces visites sachant que la DEA le traquait, et donc il n'est pas complétement invraisemblable - même si en fin de compte improbable - qu'il ait véritablement séjourné dans le barrio Luis Fanor Hernández.

Mais bien plus que la véracité - ou non - de cette histoire, c'est surtout le fait de ne jamais l'avoir entendue auparavant qui m'a le plus troublé. Je me suis demandé si j'étais peut-être moins bon ethnologue que je ne le croyais, ou bien si je commençais à perdre la main... En 2014, cela faisait dix-huit ans que j'effectuais mes recherches longitudinales sur le gang et le trafic de la drogue dans le quartier, je pensais donc bien connaître leur historique et leur développement, et que rien d'aussi bizarre et exotique que le séjour putatif de Pablo Escobar dans le barrio aurait échappé à mon attention... Cette histoire souligne aussi que la recherche est inévitablement une activité imparfaite et que l'ethnologue, même s'il est plus proche du contexte et du phénomène étudié que beaucoup d'autres, reste néanmoins toujours allochtone à ce contexte. La nature même de l'ethnographie, et en particulier son autojustification basée sur la proximité prolongée, fait qu'il devient très facile de commencer à assumer une certaine omniscience au fur et à mesure que nous accumulons une quantité conséquente de connaissances, et ceci surtout dans le cadre d'études longitudinales effectuées de manière répétées sur le long terme. De ce point de vue, le fait que je ne sache rien de l'histoire de Pablo Escobar dans le barrio apparait moins comme un échec et plus comme un avertissement salutaire. Au-delà de me fournir une anecdote inhabituelle et plutôt comique, elle me servira méthodologiquement dans la mesure où je ne compte pas arrêter mes recherches ethnographiques dans le barrio Luis Fanor Hernández de sitôt, et que je suis maintenant beaucoup plus conscient que je dois constamment garder une vigilance réflexive. Je ne peux pas nécessairement assumer que ce que j'ai appris dans le passé à propos du gang et du trafic de drogue en constitue un panorama complet et immuable. Il est clair que mes recherches futures me réserveront énormément de surprises tant par rapport aux limites de mes efforts passés qu’à de nouveaux développements.

Pour conclure, je voudrais revenir sur le titre de mon article. Pourquoi appeler à une ethnographie délinquante ? Le mot « délinquance » de nos jours s'utilise surtout pour parler de délit et de criminalité, mais étymologiquement, il dérive du latin "delinquere », qui veux plutôt dire «transgresser ». Si nous reprenons le sens original du terme, nous pouvons distinguer trois formes différentes mais liées d'ethnographie délinquante : (1) l'ethnographie de la délinquance ; (2) l'ethnologue délinquant ; (3) la délinquance de l'ethnographie. La 
première, qui se revendiquerait plus de l'usage contemporain du terme « délinquance », concerne une ethnographie préoccupée par la délinquance et les phénomènes qui y sont associés, tels les gangs. Celle-ci implique clairement des dilemmes tant pratiques que moraux, mais qui ne sont pas insurmontables, comme le montre la riche littérature ethnographique à propos des gangs ${ }^{49}$, et, surtout, qui ne sont pas une raison pour ne pas faire de la recherche sur ces phénomènes.

La deuxième forme d'ethnographie délinquante, celle de l'ethnologue délinquant, combine les associations contemporaines et originales du terme "délinquance », se référant à l'ethnologue qui s'associe à un gang - ou bien à une autre forme de criminalité - et commet des écarts aux règles et normes conventionnelles tant légales que de bienséance afin de pouvoir étudier le phénomène de manière ethnographique. En même temps, ceci présuppose des notions d'éthique de moralité fixes, alors que mes expériences au Nicaragua suggèrent que ces questions sont très souvent situationnelles 50 . Il est bien évidemment plus facile d'établir des principes et des lignes éthiques sans avoir à se préoccuper des particularités du contexte de recherche. Mais, à un moment où des cadres éthiques et des protocoles d'intégrité inspirés par les sciences naturelles sont de plus en plus renforcés et imposés dans les universités partout dans le monde ${ }^{51}$, il est important d'entamer une réflexion significative et réflexive concernant la question pour la recherche ethnographique, car celle-ci est très différente de la recherche scientifique telle que mise en ouvre par les sciences naturelles. Une question particulièrement brulante à cet égard concerne la protection des sources d'information, qui dans les sciences naturelles a surtout à faire avec la poursuite de brevets et autres moyens de faire financièrement fructifier la recherche, alors que pour les sciences sociales, et plus particulièrement l'ethnographie, elle concerne beaucoup plus le fait de s'assurer qu'aucune source d'information ne souffrira de conséquences négatives dues à la recherche. Que faire quand cela implique de protéger des individus ayant commis des crimes, comme c'est souvent le cas dans le contexte des recherches ethnographiques sur les gangs ? C'est une question peu abordée, en partie parce qu'elle peut clairement impliquer une complicité du chercheur ou de la chercheuse (le journalisme pourrait peut-être offrir des pistes de pratiques à mettre en œuvre à cet égard).

49. Decker S. H. et D. Pyrooz, «Contemporary Gang Ethnographies », in Cullen F. T. et P. Wilcox (eds.), The Oxford Handbook of Criminological Theory, Oxford, Oxford University Press, 2011, pp. 274-293 ; Rodgers D. et J. M. Hazen, "Introduction: Gangs in a Global Comparative Perspective ", in Hazen J. M. et D. Rodgers (eds.), Global Gangs: Street Violence across the World, Minneapolis, University of Minnesota Press, 2014, pp. 1-25.

50. Rodgers D., "Joining the gang...», op. cit.

51. Hellman J., "New Challenges for Fieldworkers in Latin American and Caribbean Studies ", European Review of Latin American and Caribbean Studies, $\mathrm{n}^{\circ}$ 100, 2015, pp. 99-110. 
Finalement, la troisième forme d'ethnographie délinquante, celle de la délinquance de l'ethnographie, correspond au processus ethnographique même, et à sa manière de s'imposer par rapport à l'ethnologue, de constamment surprendre, de transformer les préjugés et les idées préconçus. À un certain niveau, cela correspond au fondement même de la recherche; plutôt que d'essayer d'adapter le monde à nos cadres de recherche, il est important que ceux-ci soient adaptables à la réalité et que nous puissions être surpris et changer d'avis. Ceci est d'autant plus d'important à une époque où le financement de la recherche cherche de plus en plus à savoir avant de débourser ses ressources quels seront les résultats des recherches proposées, à quel agenda il correspond, comment le classifier. Il n'est pas surprenant qu'il y ait de moins en moins de recherches ouvertes, fondamentales, véritablement transformatrices. Vu de cette perspective, cette dernière forme de délinquance, celle de la transgression épistémologique et méthodologique, reste peut-être la plus grande force de l'approche ethnographique, car elle force une ouverture d'esprit qui ne peut être que salutaire pour la recherche, et ce d'autant plus quand celle-ci concerne les gangs, car elle permet au chercheur ou à la chercheuse de transcender les stéréotypes et préconceptions qui les entourent. 